\title{
Assimetrias laterais no movimento de chute e rendimento no futebol e no futsal
}

\author{
F.A. Barbieri, L.T.B. Gobbi
}

\begin{abstract}
Para proficiência no futebol e no futsal é necessário que o atleta apresente similar rendimento entre os membros contralaterais. No entanto, poucos atletas atingem este nível de comportamento. O objetivo deste estudo foi revisar na literatura as assimetrias laterais presentes no chute no futebol e no futsal e quais seus efeitos no rendimento. O estudo utilizou uma revisão sistemática para responder aos questionamentos. Os resultados evidenciaram as assimetrias entre os membros contralaterais com relação à habilidade de chutar, sendo necessário treinamento adequado e igualitário entre os lados para desenvolver similarmente os membros. $\mathrm{E}$ mais, a similaridade deve ser no nível mais alto, ou seja, no nível de execução do membro dominante. Ainda, no treinamento deve-se dar maior ênfase aos aspectos cinemáticos do chute do que aos aspectos relacionados à força, tanto do membro de chute como do membro de suporte. Conclui-se que as assimetrias entre os lados nos chutes existem e prejudicam o rendimento do atleta durante o jogo, mas essa questão ainda não foi solucionada, necessitando de mais estudos com relação ao treinamento e às diferenças entre os lados.
\end{abstract}

Palavras-chave: assimetria, rendimento, cinemática, futebol, futsal

\section{Lateral asymmetries in the kick movement and the soccer and futsal performance}

For proficiency, on soccer and futsal, is necessary that the athlete has similar performance between contralateral limbs. However, few athletes show this level of behavior. The aim of this study was to review in the literature the side asymmetry on soccer and futsal kick and what its effects on performance. The study used a systematic review to answer the questions. The results showed asymmetries between

Fabio Augusto Barbieri. Departamento de Educação Física, UNESP - Universidade Estadual Paulista, LEPLO - Laboratório de Estudos da Postura e da Locomoção, Departamento de Educação Física São Paulo - Brasil. UFSCAR - Departamento de Educação Física e Motricidade Humana. Lilian Teresa Bucken Gobbi. Departamento de Educação Física, UNESP - Universidade Estadual Paulista, LEPLO - Laboratório de Estudos da Postura e da Locomoção, Departamento de Educação Física - São Paulo - Brasil.

Endereço para correspondência: Lilian Teresa Bucken Gobbi, UNESP - Rio Claro - Departamento de Educação Física - LEPLO - Laboratório de Estudos da Postura e Locomoção - Avenida 24-A, 1515 Bela Vista - CEP: 13506-900 - Rio Claro/SP, Brasil. E-mail: lilian.gobbi@pq.cnpq.br 
34 | Assimetrias laterais no movimento de chute e rendimento no futebol e no futsal

contralateral limbs with respect to the ability to kick, requiring proper and equality training between the sides to develop similarly limbs. Moreover, the similarity should be at the highest level, in other words, on performance level of the dominant limb. In the training, greater emphasis to aspects of the kick kinematic than the aspects related to the strength should be performed as much the kick limb as the support limb. We conclude that the asymmetries between the sides in the kicks affect the athletes' performance during the game, but this issue is not yet solved, requiring further studies with respect to training and the differences between the sides.

Key words: asymmetry, performance, kinematics, soccer, futsal

As assimetrias laterais estão presentes em quase todos os movimentos que realizamos diariamente. Assimetria lateral é conhecida como a diferença na capacidade de controle entre os membros homólogos do lado direito e esquerdo do corpo (Haywood \& Getchell, 2004). As assimetrias ficam mais evidentes nas ações esportivas, pois dificilmente os atletas apresentam rendimento semelhante entre os lados. As diferenciações entre os lados ocorrem tanto na preferência quanto no desempenho. Preferência é a predisposição em escolher membros ou órgãos sensoriais de um lado ou de outro do corpo em diferentes tarefas. Desempenho se refere à diferença na capacidade de controle em diferentes aspectos da motricidade, tais como precisão, velocidade de execução, coordenação e latência para iniciar movimentos (Teixeira, 2006).

A ideia de assimetria lateral advém da lateralidade humana. A lateralidade é um conceito abrangente, que envolve diferentes aspectos dos seres vivos relativos aos hemisférios direito e esquerdo do corpo (Teixeira, 2006), como por exemplo, o cognitivo - referência da linha sagital mediana - e a estrutura física - diferenças entre os lados do corpo. A dominância cerebral - existência de um hemisfério cerebral dominante para determinadas funções (Kandel, Schwartz \& Jessell, 1991) , gera assimetrias na capacidade de desempenho entre os dois lados do corpo. A diferenciação funcional dos hemisférios cerebrais parece ser uma das genitoras das diferenças entre o membro dominante e o membro não dominante (Gabbard \& Hart, 1996; Sadeghi et al., 2000). Esta diferenciação advém de fatores genéticos devido às distinções estruturais no sistema nervoso central (Bryden, 1990; Kandel, Schwartz \& Jessell, 1991). O hemisfério cerebral esquerdo é especializado em funções de linguagem, pensamento analítico, processamento seriado e controle seqüenciado de atos motores. Já o hemisfério cerebral direito é adaptado para pensamento integrativo, processamento paralelo e análise de relações espaciais (Kandel, Schwartz \& Jessell, 1991). Para pessoas destras, o principal responsável 
por processamento paralelo e percepção de aspectos espaciais do ambiente é o hemisfério cerebral direito, enquanto o hemisfério esquerdo desempenha o papel principal no seqüenciamento e temporização dos movimentos (Goodale, 1990). Este formato de controle cerebral faz com que ambos os hemisférios estejam ativos durante o planejamento e a execução do movimento.

Além de aspectos genéticos, fatores ambientais também podem influenciar na preferência por um dos membros. Ashton (1982) indicou que os componentes ambientais respondem por 80-90\% na definição do membro preferido. Experiências do indivíduo durante a vida possuem papel importante no estabelecimento de preferências e assimetrias laterais (Teixeira, 2006). Para a assimetria de desempenho relacionada à prática esportiva, a quantidade de prática específica com cada membro tem efeito na determinação do membro preferido (Gobbi, Secco \& Marins, 2001; Teixeira, Silva \& Carvalho, 2003).

A determinação do membro preferido é um aspecto importante quando a assimetria entre os membros contralaterais é estudada. Dois métodos têm sido empregados para determinar o membro dominante (Oldfield, 1971; Coren, 1993; Teixeira, 2006): a) observação efetiva de uso do membro: pede-se para o indivíduo realizar tarefas motoras e é registrado qual membro foi utilizado. Comummente a mesma tarefa é realizada mais de uma vez para confirmar a consistência de uso; e, b) inventários respondidos pelo próprio indivíduo avaliado: a tarefa não é realizada fisicamente, mas o indivíduo indica qual membro utilizaria para realizar a tarefa motora específica.

No entanto, para determinar a preferência pedal é mais recomendada a observação efetiva do uso do membro para garantir maior fidedignidade e para analisar o papel que cada membro desempenha na ação. Diferentemente da preferência manual, a preferência pedal é definida de acordo com o papel do membro na tarefa (Sadeghi et al., 2000; Teixeira, Silva \& Carvalho, 2003), já que um membro é dominante para determinada tarefa enquanto para outra é o seu membro contralateral. Mediante a isso, o teste prático de realizar um chute com objetivo de acertar um alvo é a avaliação mais utilizada para determinar a preferência pedal (Chapman, Chapman \& Allen, 1997; Gabbard \& Hart, 1996; Porac e Coren, 1981).

Entretanto, alguns autores definem o membro preferido como o membro utilizado para a manipulação de um objeto ou para iniciar um movimento e não dominante como o membro que auxilia o membro dominante (Coren, 1993; Gabbard \& Hart, 1996). Esta definição é compreensível para tarefas unilaterais, já que apenas um 
dos membros é utilizado na manipulação, mas para o caso de tarefas bilaterais, como é o caso do chute, ela não se aplica. Em ações bilaterais sempre ocorre a perspectiva de um membro ser dominante para o contato com a bola e o outro dominante para o suporte ao movimento. Desta forma, não existe membro inferior dominante, pois um dos membros é usado para suporte corporal enquanto o outro é utilizado para a ação (Previc, 1991). Para as pessoas ditas destras, o membro direito é dominante para a realização do chute - membro de chute - e não dominante para o suporte e estabilização do corpo quando o chute é realizado com o membro esquerdo - membro de suporte (Barbieri et al., 2008a,b). Para as pessoas ditas sinistras, ocorre o mesmo, mas de maneira inversa, sendo o membro esquerdo dominante para a realização do chute (Barbieri et al., 2008a,b). Esta definição da preferência pedal de acordo com o papel na tarefa é freqüentemente apresentada através da assimetria funcional e soluciona teoricamente dois principais objetivos da locomoção humana: estabilidade e velocidade (Gentry \& Gabbard, 1995).

Muitos estudos comprovam que a maioria das pessoas é destra para a realização da ação e sinistra para o suporte do corpo (Gabbard \& Hart, 1996; Gentry \& Gabbard, 1995; Hart \& Gabbard, 1997; Sadeghi et al., 2000; Teixeira, 2006). Entretanto, essa tendência nem sempre é confirmada (Gobbi, Secco \& Marins, 2001). No caso do futsal e do futebol, pesquisas evidenciam que a maior parte dos jogadores são destros para as ações durante os jogos (Carey et al., 2001; Starosta \& Bergier, 1997). Aproximadamente $79 \%$ dos atletas são destros para o membro de chute e a maioria dos atletas não usa o membro não preferido durante as ações do jogo, ou quando o fazem só ocorre em situações consideradas de fácil ação (Carey et al., 2001). Atletas ambidestros, neste caso, podem ter vantagens durante a partida (Carey et al., 2001; Starosta, 1993), pois estes esportistas utilizam estratégias diferentes dos atletas destros e sinistros. Ainda, as equipes que apresentam jogadores ambidestros têm maiores chances de vencer uma partida e também maiores possibilidades técnicas e táticas durante a partida (Carey et al., 2001; Hart \& Gabbard, 1997).

A pouca utilização do membro não dominante durante a partida pode estar relacionada à assimetria na preparação técnica dos atletas (Starosta \& Bergier, 1997). Como o membro não dominante no papel de ação só ocorre em situações de baixa exigência há prejuízo no rendimento dos jogadores e da equipe durante a partida. No contexto do futsal e do futebol, onde a complexidade das ações é alta, atletas e equipe seriam beneficiadas se os membros pudessem trocar de papel (ação e suporte) de acordo com a situação. Com isso, o objetivo deste estudo foi 
revisar na literatura as assimetrias laterais presentes no movimento de chute no futebol e futsal, com a bola em posição estacionária e em deslocamento, e verificar seus efeitos no rendimento. Primeiramente foram analisadas as assimetrias no desempenho e na velocidade da bola entre os chutes com o membro dominante e não dominante, apontando fatores responsáveis pelas diferenças entre os lados. Posteriormente, a influência dos aspectos cinemáticos do movimento no rendimento do chute com o membro dominante e com o membro não dominante, analisando o papel de cada separadamente e verificando suas influências no rendimento.

\section{Metodologia}

O estudo caracteriza-se como uma revisão da literatura. Foram selecionados estudos que definiam as assimetrias laterais e trabalhos que tinham como objetivo a análise dessas assimetrias durante o movimento de chute. Os artigos foram criteriosamente escolhidos utilizando as bases de dados PubMed e Scielo pelo ano de publicação e dando preferência a estudos que faziam referência ao foco de estudo, com critérios de definição de assimetria bem estabelecidos.

Para análise dos resultados encontrados, as assimetrias laterais foram agrupadas em dois aspectos importantes: desempenho e velocidade da bola; e aspectos cinemáticos do chute.

\section{Desenvolvimento}

A assimetria no desempenho e na velocidade da bola nos chutes

Os dados encontrados nos estudos demonstram assimetria tanto para o desempenho quanto para a velocidade da bola nos chutes com a bola parada (chutes no qual a bola se encontra em posição estacionária, como por exemplo, cobranças de faltas e penalidades).

A Tabela 1 apresenta a velocidade da bola observada nos chutes com o membro dominante e não dominante com a bola parada para jogadores da categoria adulta. Nota-se que o membro dominante apresenta velocidade do chute significantemente superior ao membro não dominante em todos os estudos apresentados. 
38 | Assimetrias laterais no movimento de chute e rendimento no futebol e no futsal

Tabela 1. Velocidade da bola nos chutes com a bola parada realizado com o membro dominante e membro não dominante.

\begin{tabular}{cccc}
\hline AUTORES & MEMBRO & MEMBRO NÃO & $P$ \\
& DOMINANTE $\left(\mathrm{m} \cdot \mathrm{s}^{-1}\right)$ & DOMINANTE $\left(\mathrm{m} \cdot \mathrm{s}^{-1}\right)$ & \\
\hline McLean \& Tumilty (1993) & $21,9 \pm 1,6$ & $18,3 \pm 2,2$ & $<0,05$ \\
Mognoni et al. (1994) & $23,6 \pm 2,5$ & $21,4 \pm 2,6$ & $<0,05$ \\
Barfield (1995) & $26,4 \pm 2,1$ & $24,3 \pm 2,0$ & $<0,01$ \\
Patritti (1997) & $23,0 \pm 1,3$ & $21,2 \pm 1,3$ & $<0,001$ \\
Dörge et al. (2002) & $24,7 \pm 2,5$ & $21,5 \pm 2,0$ & $<0,05$ \\
Barfield et al. (2002) & $25,3 \pm 1,5$ & $23,6 \pm 1,6$ & $<0,05$ \\
Nunome et al. (2006) & $32,1 \pm 1,6$ & $27,1 \pm 1,6$ & $<0,05$ \\
Barbieri (2007) & $24,2 \pm 2,2$ & $21,6 \pm 2,3$ & $<0,001$ \\
\hline
\end{tabular}

A diferença na velocidade da bola entre os lados prejudica o rendimento do jogador durante a partida, já que este não consegue desenvolver um chute com similar velocidade e potência entre os membros contralaterais. Poucos atletas conseguem eficiência semelhante para a velocidade da bola no chutes, o que para a dinâmica do jogo é ruim, já que nem sempre existe a possibilidade de uso do membro dominante durante a ação. Com esta perspectiva fica evidente o prejuízo para a equipe e para o atleta, já que em uma ação que seja necessário utilizar o membro não dominante para finalizar a gol o rendimento é diminuído.

A utilização de ambos os membros similarmente aumenta o rendimento do atleta. Em desportos que ocorrem em alta velocidade de movimentação e com marcação sempre próxima, como no futebol e no futsal, não há tempo para que em todos os momentos o jogador prepare a bola e posicione o corpo para utilizar o membro dominante. Este contexto é mais evidente no futsal, uma vez que algumas características são distintas entre os esportes como o espaço de jogo, o número de jogadores em cada equipe e a dimensão e pressão da bola, fazendo com que o atleta tenha menos tempo para a realização da ação e necessite mais do membro não dominante. No futsal, a dinâmica do jogo é mais intensa, o que faz com que os jogadores atuem por toda a extensão da quadra; enquanto no futebol, há posicionamento específico dos atletas em campo. Com isso, o membro não dominante é menos exigido para algumas posições no futebol. No entanto, é necessário ressaltar que o uso dos dois lados de forma similar auxilia bastante no rendimento do jogador e sua proficiência no jogo é atingida quando há simetria (Teixeira, Silva \& Carvalho, 2003). 
A assimetria na velocidade da bola não aparece apenas no chute com a bola parada, mas também no chute com a bola em deslocamento no futsal (chutes que resultam da preparação da bola por um atleta para seu companheiro realizar a finalização com a bola em deslocamento), também com favorecimento do membro dominante em relação ao membro não dominante $\left(23,88 \pm 2,7 \mathrm{~m} \cdot \mathrm{s}^{-1}\right.$ e $21,42 \pm 2,25$ $\mathrm{m} \cdot \mathrm{s}^{-1}$, respectivamente) (Barbieri, 2007). Neste contexto, as assimetrias no chute com a bola em deslocamento são piores para o rendimento do jogador, uma vez que $69 \%$ das finalizações no futsal é decorrente de chutes com a bola em deslocamento, sendo bem mais utilizada que o chute com a bola parada (Ferreira, 1999).

A eficiência do chute com a bola em deslocamento é baixa (Ferreira, 1999), talvez devido à assimetria. Diferentemente do chute com a bola parada, no chute com a bola em deslocamento é difícil o jogador conseguir preparar a bola para realizar sempre o chute com o membro dominante. Como a maioria das oportunidades de gols surge deste tipo de chute, conseqüentemente, quanto melhor o desempenho nesta ação, maiores as chances de vencer uma partida. Por outro lado, o chute com a bola parada é mais eficiente durante a partida, como em cobrança de falta e penalidade, pois sempre existe a possibilidade de utilizar o membro dominante.

É provável que a assimetria no futebol e futsal possa estar inserida no processo de preparação e treinamento, uma vez que a diferença entre os lados para a velocidade da bola não foi somente constatada entre os homens, mas também entre as mulheres praticantes. Como os homens, as mulheres apresentam chutes mais velozes com o membro dominante $(21,5 \pm 2,44 \mathrm{~m} . \mathrm{s}-1)$ que o membro não dominante $(18,9 \pm 2,05$ m.s-1) (Barfield, Kirkendall \& Yu, 2002). Assim, cuidados com as intervenções propostas durante as atividades realizadas no futebol e futsal são necessários para tentar diminuir estas assimetrias. Precauções com a transferência e interferência bilateral são importantes durante as atividades práticas, uma vez que adaptações perceptivas e neurais são necessárias durante o processo de transferência de aprendizagem, podendo causar interferências significativas (Teixeira, 2006). No entanto, não é objetivo deste estudo abordar este aspecto com relação ao movimento de chute.

A assimetria não é apenas observada na velocidade da bola, mas também é clara no desempenho dos chutes. As diferenças entre os lados no desempenho aparecem em diversos tipos de chutes. Para o chute rasteiro, considerado um chute de fácil realização, o desempenho do membro dominante é $50 \%$ maior que o do membro não dominante; enquanto para a "cavadinha", que é de difícil execução, 
não foi notado assimetria no desempenho (McLean \& Tumilty, 1993). Parece que a assimetria relacionada ao desempenho é dependente da tarefa a ser realizada, indicando diminuição nas diferenças à medida que a complexidade do movimento aumenta. Nesta mesma linha, o chute com a bola em deslocamento apresenta maior assimetria entre os lados que o chute com a bola parada (Barbieri, 2007). Quando o desempenho do chute de potência é analisado, a assimetria entre os lados é ainda mais evidenciada. Neste tipo de chute, o membro dominante apresenta desempenho três vezes melhor que o membro não dominante (Barbieri et al., 2005; Barbieri, Lima Junior \& Gobbi, 2006; Barbieri, 2007; Teixeira, Silva \& Carvalho, 2003), o que concorda com a Lei de Fitts (1954), uma vez que a relação entre potência e precisão é negativamente proporcional.

Para diminuir as assimetrias apresentadas para a velocidade da bola e para o desempenho, o treinamento similar entre os lados parece ser importante. Estudos demonstram que o treinamento similar entre os lados apresenta melhoras no desempenho do lado não dominante (Haaland \& Hoff, 2003; Teixeira, Silva \& Carvalho, 2003). No entanto, o desempenho do membro dominante ainda parece maior mesmo após o período de treinamento. Este procedimento tem sido aplicado por período curto de tempo (2 ou 3 meses), enquanto a especificidade pelo membro dominante vem ocorrendo por longo período (anos) da preparação. Por isso, carga e intensidade semelhante entre os lados devem ocorrer desde o princípio do treinamento do futebol e futsal. Ainda, o rendimento a ser atingido deve ser equiparado ao mais alto nível, ao membro dominante. Desta forma, a preparação bilateral do atleta de futebol e futsal deve ocorrer durante todo o período de treinamento.

\section{Aspectos cinemáticos das assimetrias nos chutes}

As diferenças encontradas entre os lados para o desempenho e para a velocidade da bola são principalmente influenciadas por diferenças nos parâmetros cinemáticos (deslocamento, velocidade linear e angular, aceleração, entre outros) do movimento do membro de chute e do membro de suporte. No entanto, a maioria dos estudos analisa o membro de chute, negligenciando o estudo do membro de suporte. Apesar disso, este membro tem relevante importância durante o movimento de chute no futebol e futsal. Além de sustentar o corpo, o posicionamento do membro de suporte afeta a precisão do movimento (Barbieri et al., 2008a). Para melhorar a eficiência do chute, é necessário atenção ao membro de suporte, para que este auxilie de forma adequada o membro de chute (Carey et al., 2001). 
Com relação aos aspectos cinemáticos do movimento do membro de chute, a velocidade da bola e o desempenho dos chutes com o membro dominante estão intimamente relacionados com as diferenças cinemáticas e mecânicas posicionamento, deslocamento, velocidade e aceleração dos segmentos e articulações - entre os lados que favorecem o membro dominante (Barbieri et al., 2005; Barbieri, 2007; Barfield, 1995; McLean \& Tumilty, 1993). As assimetrias notadas no padrão de movimento de chute aparecem principalmente em dois momentos do ciclo de movimento: no início e no contato com a bola (Barbieri et al., 2005; Barbieri, 2007). O início do movimento é importante, pois correções posteriores são difíceis de ocorrer; de forma que o começo inadequado compromete tanto o posicionamento ideal para o contato com a bola como a meta do chute. O momento de contato com a bola define o rendimento do chute; assim, a posição das articulações no momento de contato com a bola é de fundamental importância para o resultado final do chute (Mognoni et al., 1994). Por isso, durante a realização da ação de chute, a atenção do executante deve estar dirigida para estes dois momentos, a fim de sanar deficiências no movimento e aumentar o rendimento do chute.

Nos chutes com o membro dominante, a velocidade angular do joelho e da perna e a velocidade linear do pé de chute no momento de impacto com a bola são maiores em comparação ao membro não dominante. Estes parâmetros cinemáticos contribuem para o rendimento do chute (Barbieri, 2007; Dörge et al., 2002; Patritti, 1997), que pode ser evidenciado pela velocidade linear do pé de chute. A maior velocidade do lado dominante em relação ao não dominante não ocorre apenas no

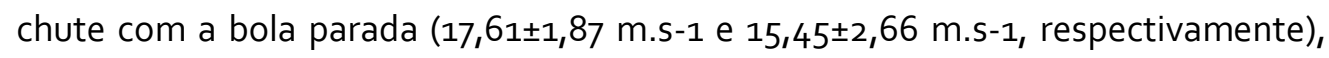
mas também no chute com a bola em deslocamento $(17,25 \pm 2,26 \mathrm{~m} . \mathrm{s}-1$ e $14,56 \pm 2,57 m .5-1$, respectivamente) (Barbieri, 2007).

No entanto, para que a velocidade do pé no momento de contato com a bola seja adequada é preciso ter alta relação entre os segmentos do mesmo lado. O membro não dominante apresenta pior relação intersegmentar devido à menor habilidade e à maior variabilidade deste lado (Barbieri et al., 2008a). Se esta relação é ruim, o executante não consegue transferir o movimento e a velocidade de um segmento para outro, prejudicando a produção da velocidade apropriada do pé para o contato com a bola. A maior consistência no movimento, ou seja, menor variabilidade do membro dominante é relevante para a eficácia do chute e pode causar deficiências no rendimento com o membro não dominante (Barbieri et al., 2008a). Devido à maior variabilidade do membro não dominante, nem sempre o executante consegue produzir a velocidade esperada no chute para este lado, mesmo quando 
maiores velocidades lineares da articulação do tornozelo e do pé são produzidas em relação ao lado dominante (Barbieri et al., 2008a).

A relação intersegmentar é mais efetiva no lado dominante; entretanto, a força muscular parece ser um fator de pouca relevância. Estudos discordam com relação ao rendimento do chute ser dependente de maior força muscular para o membro não dominante ou para o membro dominante (Amato et al., 2001; Capranica et al., 1992; Goulart, Dias \& Altimari, 2007; Rahnama, Lees \& Bambaecichi, 2005; Zakas, 2005, 2006). Além disso, níveis elevados de força sem boa relação intersegmentar não são efetivos. Com isso, somente o treinamento de força muscular não trará benefícios para o rendimento do chute, sendo necessário desenvolver a técnica de movimento para transmitir a velocidade dos segmentos e a força para a bola por meio da transferência intersegmentar. No entanto, o treinamento de força muscular pode ser interessante em determinadas fases da preparação. Este tipo de treinamento deve sempre ocorrer juntamente com o treinamento da técnica do movimento, já que outros fatores, como a habilidade, o controle do movimento, a interação intersegmentar, a postura, etc., podem estar envolvidos. A prescrição de atividades que estimulem o uso do membro não dominante pode auxiliar no desempenho dos atletas, sendo interessante o estudo da periodização e da quantidade de carga para cada lado.

Em relação aos aspectos cinemáticos do membro de suporte, assimetrias entre os lados foram observados na corrida de aproximação (Barbieri, Lima Junior \& Gobbi, 2006). Variáveis como a largura e a amplitude do penúltimo passo e a posição do pé em relação à bola são aspectos distintos entre os lados, enquanto a velocidade da corrida de aproximação e a amplitude do último passo não apresentam relevância para um chute com bom desempenho e alta velocidade da bola entre os lados (Barbieri, Lima Junior \& Gobbi, 2006). Durante a corrida de aproximação do chute com o membro não dominante, o executante prefere melhorar o equilíbrio e a postura para tentar aumentar o resultado final do chute, ao invés de procurar aumentar a velocidade da corrida de aproximação, que é um fator importante para a velocidade do chute (Dörge et al., 2002).

Como o membro de chute, o membro de suporte também apresenta maior variabilidade de movimento articular para o lado não dominante do que para o membro dominante, que apresentaram maior consistência de movimento, principalmente na fase de apoio (Barbieri et al., 2008b). Diferenças no deslocamento e na velocidade angular no membro de suporte entre os lados indicam que o posicionamento inadequado do pé de suporte pode resultar em 
queda do rendimento no chute (Barbieri, 2007). Estas assimetrias no membro de suporte podem prejudicar o movimento de alavanca do membro de chute e o movimento contrário realizado pelo membro suporte, uma vez que estes movimentos são dirigidos pelo quadril e esta articulação é a mais distinta entre os lados para o membro de suporte (Barbieri et al., 2008b).

Assim, tanto o membro de chute como o membro de suporte influenciam o rendimento do chute para os aspectos de desempenho e da velocidade da bola. Por isso, durante a execução do chute e o treinamento atenção similar deve ser dada para o membro de chute e de suporte a fim de aumentar o rendimento do chute.

\section{Conclusões}

A partir da presente revisão foi possível evidenciar as assimetrias entre os membros contralaterais com relação à habilidade de chutar. Desta maneira, é importante determinar como diminuir a diferença entre os lados, já que para se atingir a proficiência no futebol e futsal é indispensável rendimento similar entre os lados. $\mathrm{E}$ mais, a similaridade deve ser no nível mais alto, ou seja, no nível de execução do membro dominante.

Com isso, o treinamento adequado e igualitário entre os lados é essencial para desenvolver similarmente os membros contralaterais. No entanto, o que ocorre no futebol e futsal é uma preocupação excessiva em treinar o membro dominante, negligenciando o membro não dominante. A necessidade de resultados imediatos na formação pode ser um dos fatores do treinamento acentuado do membro dominante, pois este trará melhor rendimento para os atletas apenas nas categorias de base do futebol e futsal. Quando este jogador atingir o alto nível, haverá a necessidade do uso habilidoso do membro não dominante, já que o jogo é mais dinâmico e os atletas mais equilibrados técnica, tática e fisicamente. Assim, os jogadores terão dificuldades em progredir seu rendimento, enquanto jogadores que tiveram uma formação mais completa e diversificada obterão melhores rendimentos. Nesta perspectiva, o nível de habilidade do lado dominante é restrito pelo nível de habilidade do lado não dominante e o desenvolvimento de habilidades do membro não dominante cria mais possibilidades para ações durante a prática do futebol e futsal (Haaland \& Hoff, 2003). Ainda, a partir dos estudos revisados pode-se afirmar que durante o treinamento deve-se dar maior ênfase aos aspectos cinemáticos do chute - posicionamento da articulação, transferência intersegmentar, velocidade linear e angular - do que aos aspectos relacionados à força. 
A atenção para o membro de suporte nos treinamentos deve ser a mesma dispensada para o membro de chute, pois o correto posicionamento do membro de suporte auxilia o movimento e facilitar o posicionamento das articulações do membro para o chute. Estes cuidados beneficiam a velocidade da bola e o desempenho nos chutes.

Com adequada intervenção no movimento dos jogadores, ambos os membros poderão ter ações habilidosas que são marcadas pela pouca variabilidade do movimento (Connolly, 1997; Tani, 2000). Para que este fato se efetive, deve ser dada ênfase similar para os dois membros inferiores durante o refinamento do futsal (Barbieri et al., 2008a). O alto rendimento do atleta com ambos os membros facilita as ações durante a partida, pois aumenta as possibilidades de ações tácticas e técnicas. Além disso, para rendimento de sucesso nos chutes, o atleta necessita apresentar bom equilíbrio e controle postural (Sforza et al., 1997), que também serão beneficiados com o aumento do treinamento com o membro não dominante.

Conclui-se, dessa forma, que as assimetrias entre os lados nos chutes existem e prejudicam o rendimento do atleta durante o jogo, mas essa questão ainda não foi solucionada, necessitando de mais estudos com relação ao treinamento e às diferenças entre os lados. Estudos longitudinais com acompanhamento do processo de refinamento da habilidade de chutar e do treinamento visando a simetria intermembros são recomendados.

\section{Agradecimentos}

Os autores do estudo gostariam de agradecer à CAPES pelo apoio financeiro.

\section{Bibliografia}

Amato, M., Lemoine, F., Gonzales, J., Schmidt, C., Afriat, P., \& Bernard, P.L. (2001). Influence of age and physical activity on isokinetic characteristics of hamstring and quadriceps muscles of young gymnasts and soccer players. Annales de Readaptation et de Medecine Physique, 44(9), 581-590.

Asthon, G.C. (1982). Handedness: An alternative hypotesis. Behavior Genetics, 12(2), 125147.

Barbieri, F.A., Moura, F.A., Lima Júnior, R., Wisiak, M., Santiago, P.R.P., Thomaz, T., \& Cunha, S.A. (2005). Padrão cinemático do membro de suporte no chute realizado com os membros dominante e não dominante no futsal. Lecturas: Educacion Física y Deportes, 10(90), 1-11.

Barbieri, F.A. (2007). O chute com o membro dominante e não dominante realizado com a bola parada e em deslocamento no futsal. Dissertação de Mestrado em Ciências da 
Motricidade não publicada, Rio Claro: Instituto de Biociências, Universidade Estadual Paulista.

Barbieri, F.A., Lima Junior, R.S., \& Gobbi, L.T.B. (2006). Aspectos da corrida de aproximação entre o chute realizado com o membro dominante e não dominante. Revista Motricidade, 2(3), 80-90.

Barbieri, F.A., Santiago, P.R.P., Gobbi, L.T.B., \& Cunha, A.S. (2008a). Diferenças entre o chute realizado com o membro dominante e não-dominante no futsal: Variabilidade, velocidade linear das articulações, velocidade da bola e desempenho. Revista Brasileira de Ciências do Esporte, 29(2), 129-146.

Barbieri, F.A., Santiago, P.R.P., Gobbi, L.T.B., \& Cunha, A.S. (2008b). Análise cinemática da variabilidade do membro de suporte dominante e não dominante durante o chute no futsal. Revista Portuguesa de Ciências do Desporto, 8(1), 68-76.

Barfield, W.R. (1995). Effects of selected kinematics and kinetic variables on instep kicking with dominant and non-dominant limbs. Journal of Human Movement Studies, 29, 251272

Barfield, W.R., Kirkendall, D.T., \& Yu, B. (2002). Kinematic instep kicking differences between elite female and male soccer players. Journal of Sports Science and Medicine, 1(3), 72-79.

Bryden, M.P. (1990). Choosing sides: The left and right of the normal brain. Canadian Psychology, 31, 297-309.

Capranica, L., Cama, G., Fanton, F., Tessitore, A., \& Figura, F. (1992). Force and power of preferred and non-preferred leg in young soccer players. Journal of Sports Medicine and Physical Fitness, 32(4), 358-363.

Carey, D.P., Smith, G., Smith, D.T., Shepherd, J.W., Skriver, J., Ord, L., \& Rutland, A. (2001). Footedness in world soccer: an analysis of France ' 98 . Journal of Sports Science, 19, 855864 .

Chapman, J.P., Chapman, L.J., \& Allen, J.J. (1997). The measurement of foot preference. Neuropsychologia, 25(3), 579-584.

Connolly, K.J. (1997). The nature of motor skill development. Human Movement Studies, 3, 128-143.

Coren, S. (1993). The lateral preference inventory for measurement of handedness, footedness, eyedness and earedness: Norms for young adults. Bulletin of the Psychosomatic Society, 31(1), 1-3.

Dörge, H.C., Bullandersen, T., Sorensen, H., \& Simonsen, E.B. (2002). Biomechanical differences in soccer kicking with the preferred and non-preferred leg. Journal of Sports Science, 20(8), 293-299.

Ferreira, P. (1999). Caracterização da finalização em equipas de futsal. Dissertação de Mestrado em Ciências do Desporto não publicada. Maia: Instituto Superior de Maia.

Fitts, P.M. (1954). The information capacity of the human motor system in controlling the amplitude of movements. Journal of Experimental Psychology, 47, 381-391. 
46 | Assimetrias laterais no movimento de chute e rendimento no futebol e no futsal

Gabbard, C., \& Hart, S. (1996). A question of foot dominance. Journal of General Psychologic, 123(4), 289-296.

Gentry, V., \& Gabbard, C. (1995). Foot-preference behavior: A developmental perspective. Journal of General Psychology, 122(1), 37-45.

Gobbi, L.T.B., Secco, C.R., \& Marins, F.H.P. (2001). Preferência pedal: Comportamento locomotor em terreno irregular. In L.A. Teixeira (Ed.), Lateralidade e Comportamento Motor: Assimetrias laterais de desempenho e transferência interlateral de aprendizagem (pp. 225-247). São Paulo: Movimento,

Goble, D.J., Marino, G.W., \& Potvin, J.R. (1995). The influence of horizontal velocity on interlimb symmetry in normal walking. Human Movement Science, 22(3), 271-283.

Goodale, M.A. (1990). Brain asymmetries in the control of reaching. In M.A. Goodale (Ed.), Vision and Action. Amsterdam: North-holland.

Goulart, L.F., Dias, R.M.R., \& Altimari, L.R. (2007). Força isocinética de jogadores de futebol categoria sub-20: comparação entre diferentes posições de jogo. Revista Brasileira de Cineantropometria e Desempenho Humano, 9(2), 165-169.

Haaland, E., \& Hoff, J. (2003). Non-dominant leg training improves the bilateral motor performance of soccer players. Scandinavian Journal of Medicine and Science in Sports, 13(3), 179-184.

Hart, S., \& Gabbard, C. (1997). Examining the mobilizing feature of footedness. Perceptual and Motor Skills, 86, 1339-1342.

Haywood, K.M. \& Getchell, N. (2004). Desenvolvimento Motor ao Longo da Vida. Porto Alegre: Artmed.

Kandel, E.R., Schwartz, J.H. \& Jessel, T.M. (1991). Principles of Neural Science (third edition). New York: Elsevier Science.

Masuda, K., Kikuhara, N., Demura, S., Katsuta, S., Yamanaka, K. (2005). Relationship between muscle strength in various isokinetic movements and kick performance among soccer players. Journal of Sports Medicine and Physical Fitness, 45, 44-52.

McLean, B.D., \& Tumilty, D.McA. (1993). Left-right asymmetry in two types of soccer kick. British Journal of Sports Medicine, 27(4), 260-262.

Mognoni, P., Narici, V., Sirtori, D., \& Lorenzelli, F. (1994). Isokinetic torques and kicking maximal ball velocity in young soccer players. Journal of Sports Medicine and Physical Fitness, 34(4), 357-361.

Nunome, H., Ikegami, Y., Kozakai, R., Apriantono, T., \& Sano, S. (2006). Segmental dynamics of soccer instep kicking with the preferred and non-preferred leg. Journal of Sports Science, 24(5), 529-540.

Oldfield, R.C. (1971). The assessment and analysis of handedness: The Edinburgh inventory. Neuropsychologia, 9(1), 97-113.

Patritti, B.L. (1997). 3-Dimensional kinematic analysis of the maximal instep kick in soccer with the preferred and non-preferred kicking leg [Major project submitted to the Centre for Sport and Exercise Sciences]. Liverpool: John Moores University. 
Porac, C., \& Coren, S. (1981). Lateral preferences and human behavior. New York: SpringerVerlag.

Previc, F.H. (1991). A general theory concerning the prenatal origins of cerebral lateralization in humans. Psychological Review, 98, 299-334.

Rahnama, N., Lees, A., \& Bambaecichi, E. (2005). A comparison of muscle strength and flexibility between the preferred and non-preferred leg in English soccer players. Ergonomics, 48(11/14), 1568-1575.

Sadeghi, H., Allard, P., Prince, F., \& Labelle, H. (2000). Symmetric and limb in able-bodied gait: A review. Gait and Posture, 12(1), 34-45.

Sforza, C., Dugnani, S., Mauro, F., Torri, L., \& Ferrario, V. (1997). Repeatability of the football penalty: A statical evaluation by the morphological variation analysis. In $\mathrm{T}$. Reilly (Ed.), Science and Football III (pp. 240-245). London: E\&FN Spon.

Starosta, W. (1993). Symmetry and asymmetry in shooting demonstrated by elite soccer players. In T. Reilly et al. (Ed.), Science and Football II (pp. 346-355). London: E\&FN Spon.

Starosta, W., \& Bergier, J. (1997). Pattern of a sport technique in football based on the symmetry of movements. In T. Reilly (Ed.), Science and Football III (pp. 194-200). London: E\&FN Spon.

Tani, G. (2000). Variabilidade e programação motora. In J. Barbanti (Ed.), A biodinâmica do movimento humano e suas relações interdisciplinares (pp. 245-260). São Paulo: Editora Estação Liberdade.

Teixeira, L.A. (2006). Controle Motor. São Paulo: Manole.

Teixeira, L.A., Silva, M.V.M., \& Carvalho, M. (2003). Reduction of lateral asymmetries in dribbling: the role of bilateral practice. Laterality, 8(1), 53-65.

Wong, P., Chamari, K., Chaouachi, A., Mao, D.W., Wisløff, U., \& Hong, Y. (2007). Difference in plantar pressure between the preferred and non-preferred feet in four soccer-related movements. British Journal of Sports Medicine, 41, 84-92.

Zakas, A. (2005). The effect of stretching duration on the lower extremity flexibility of adolescent soccer players. Journal of Bodywork and Movement Therapies, 9, 220-225.

Zakas, A. (2006). Bilateral isokinetic peak torque of quadriceps and hamstring muscles in professional soccer players with dominance on one or both two sides. Journal of Sports Medicine and Physical Fitness, 46(1), 28-35. 